\title{
Deciphering immune mechanisms in chronic inflammatory demyelinating polyneuropathies
}

\author{
Jolien Wolbert, ${ }^{1}$ Mandy I. Cheng, ${ }^{2}$ Gerd Meyer zu Horste, ${ }^{1}$ and Maureen A. Su ${ }^{2,3}$ \\ 'Department of Neurology with Institute of Translational Neurology, University Hospital Münster, Münster, \\ Germany. ${ }^{2}$ Department of Microbiology Immunology and Medical Genetics and ${ }^{3}$ Department of Pediatrics, UCLA, \\ Los Angeles, California, USA.
}

\begin{abstract}
Chronic inflammatory demyelinating polyneuropathy (CIDP) is an autoimmune disease of the peripheral nerves that presents with either chronic progression or relapsing disease. Recent studies in samples from patients with CIDP and mouse models have delineated how defects in central (thymic) and peripheral (extrathymic) immune tolerance mechanisms can cause PNS autoimmunity. Notably, nerve parenchymal cells actively contribute to local autoimmunity and also control disease outcome. Here, we outline how emerging technologies increasingly enable an integrated view of how immune cells and PNS parenchymal cells communicate in CIDP. We also relate the known heterogeneity of clinical presentation with specific underlying mechanisms. For example, a severe subtype of CIDP with tremor is associated with pathogenic IgG4 autoantibodies against nodal and paranodal proteins. An improved understanding of pathogenic mechanisms in CIDP will form the basis for more effective mechanism-based therapies.
\end{abstract}

Authorship note: JW and MIC contributed equally to this work. CMZH and MAS are co-senior authors.

Conflict of interest: GMZH has received research support from Merck (Grant for Multiple Sclerosis Innovation). MAS has served on the external advisory board for Amgen.

Copyright: (c) 2020, American Society for Clinical Investigation.

Reference information: JCI Insight. 2020;5(3):e132411.

https://doi.org/10.1172/jci.

insight.132411.

\section{Introduction}

Chronic inflammatory demyelinating polyneuropathy (CIDP) is an autoimmune disease of the peripheral nerves and nerve roots. It is the most common chronic autoimmune neuropathy in humans and is estimated to affect up to 1 in 10,000 individuals (1). Although multiple subtypes and variants of chronic inflammatory neuropathies with diverse corresponding acronyms have been described, CIDP remains the most common form, and we specifically focus here on CIDP and CIDP models. Although the first descriptions of patients potentially originated in the 1800s, CIDP was first described as "one nosologic entity" by Peter Dyck and colleagues in 1975 (2). CIDP is characterized by symmetric loss of motor and sensory function, and diagnostic tests demonstrate electrophysiological and histological evidence of demyelination. Additional pathologic features include interstitial edema, "onion bulb" formation, which is indicative of repeated demyelination and remyelination episodes, and endoneurial inflammatory cell infiltrates composed of monocytes and lymphocytes.

For most patients, the clinical course of CIDP progresses slowly: however, one-third of patients can present with relapsing-remitting disease (3), which by definition progresses or relapses for more than eight weeks. This time-course distinguishes CIDP from acute inflammatory demyelinating polyneuropathies (AIDP) and other subtypes of Guillain-Barré syndrome (GBS), which are monophasic and usually peak within three to four weeks; although, their clinical course is also very heterogeneous. Triggered by the success of its counterpart in GBS $(4,5)$, the International CIDP Outcome Study (ICOS) was recently launched to better define CIDP heterogeneity and identify clinical and biological outcome determinants (6). This prospective, observational, multicenter cohort study promises to provide a wealth of information about the natural history of CIDP.

Unlike most other chronic neuropathies, CIDP is treatable, albeit with considerable socioeconomic implications and variable success. Current mainstays of CIDP therapy are glucocorticoids, intravenous immunoglobulin (IVIg), and plasmapheresis. With current therapies, only $11 \%$ of patients with CIDP experienced stable disease lasting more than 5 years off therapy (7). Moreover, $12 \%$ of patients had a progressive or relapsing course despite treatment and 51\% required continued treatment to prevent disease progression. Thus, more specific, effective therapies for CIDP are desirable, and understanding the underlying pathogenesis will facilitate their development. This Review will synthesize recent advances in 
understanding CIDP pathogenesis and discuss emerging technologies that hold promise in revealing new aspects of underlying disease mechanisms.

\section{Using animal models to understand CIDP}

Animal models have been instrumental in understanding the pathogenesis of chronic inflammatory neuropathies $(8,9)$. CIDP mouse models have been developed, of which multiple are on the autoimmune-prone NOD background. This predisposition for PNS autoimmunity on the NOD background is likely due to genetic loci (including the NOD-specific $\mathrm{H}-2^{27} \mathrm{MHC}$ region) that confer general susceptibility to autoimmunity (10). NOD models of CIDP include (a) NOD.Aire ${ }^{\mathrm{GW} /+}$ mice, which have a dominant loss-of-function G228W mutation in the autoimmune regulator (Aire) gene $(11,12)$; (b) NOD.ICAM1 $1^{\mathrm{tm} 1 \mathrm{Jcgr}}$ mice, which express alternative splice isoforms of the adhesion molecule ICAM1 (13); (c) NOD.B7-2 $2^{-/}$mice, which lack costimulatory molecule B7-2 (also known as CD86) (14); and (d) NOD.H-2 ${ }^{\mathrm{b}} \cdot \mathrm{PD}-1^{-/-}$mice, which express the antidiabetogenic $\mathrm{MHC}$ H-2 ${ }^{\mathrm{b}}$ haplotype and lack PD-1 $(9,15)$. These mice all display multiple features of CIDP, including spontaneous demyelination and immune cell infiltration of peripheral nerves. Importantly, PNS autoimmunity develops spontaneously in these models without the need for antigen/adjuvant immunization, mirroring the clinical observation that CIDP most often develops without antecedent triggers.

Animal models have led to the identification of tolerance mechanisms important in preventing PNS autoimmunity. Two decades ago, Gold et al. showed that, although the blood-nerve barrier (BNB) prevents cells from randomly migrating into the PNS, the peripheral nerve is under constant immune surveillance by $\mathrm{T}$ and B cells and macrophages (16). A specialized immune response takes place in the PNS that offers immune protection as well as rapid elimination of inflammation within the tissue (17). However, if immunologic tolerance is lost, these same cell types may become activated toward autoantigens expressed by the peripheral nerve cells and an autoimmune polyneuropathy may develop (18). CIDP mouse models have allowed in vivo dissection of how loss of tolerance can initiate and enhance autoimmune responses in the PNS.

\section{PNS-specific tolerance mechanisms}

Tissue autoimmunity is prevented by a series of central (thymic) and peripheral (extrathymic) immune tolerance mechanisms, which restrain the development and activation of self-reactive lymphocytes. Strong evidence exists that shows that central tolerance mediated by the Aire gene plays a major role in preventing PNS autoimmunity (Figure 1A). Within thymic epithelial cells, Aire normally induces expression of thousands of self-antigens (an "immunological mirror image" of the proteome). Recognition of these self-antigens by developing $\mathrm{T}$ cells leads to their negative selection or conversion into immunosuppressive Tregs. This core process of central tolerance prevents the release of self-reactive $\mathrm{T}$ effector cells into the periphery and subsequent development of autoimmunity. Multiple lines of evidence support Aire as critical for preventing PNS autoimmunity by upregulating expression of PNS-specific antigens in the thymus. Patients with loss-of-function AIRE mutations develop a multiorgan autoimmune syndrome that includes a CIDP-like phenotype as a component (19). Additionally, NOD mice with a dominant loss-of-function Aire G228W mutation (NOD.Aire ${ }^{\mathrm{GW} /+}$ ) spontaneously develop PNS autoimmunity that shares multiple CIDP features. Although the PNS-specific antigen myelin protein zero (abbreviated P0 or MPZ) is expressed in medullary thymic epithelial cells of WT mice, it is absent in NOD.Aire ${ }^{\mathrm{GW} /+}$ mice, suggesting that Aire normally upregulates P0 expression in the thymus to protect against PNS autoimmunity (12). Consistent with a model in which defective negative selection of P0-reactive T cells leads to PNS autoimmunity, NOD. Aire ${ }^{\mathrm{GW} /+}$ mice have increased $\mathrm{P} 0$-specific cells in the periphery and elevated $\mathrm{P} 0$-specific autoantibodies in serum (ref. 12 and Figure 1A). Together, these findings suggest that Aire plays an important role in enforcing immune tolerance toward the PNS in both humans and mice.

In addition to Aire, the adhesion and costimulatory molecule ICAM1 also plays an essential role in enforcing central tolerance toward the PNS. Although the role of ICAM1 has been extensively characterized in the peripheral immune response, studies using NOD.ICAM1 $1^{\mathrm{tm} 1 \mathrm{Jcgr}}$ mice reveal its importance in editing the $\mathrm{T}$ cell repertoire through central tolerance mechanisms. Transplantation of NOD.ICAM1 ${ }^{\mathrm{t} 1 \mathrm{Jcgr}}$ thymic stromal cells is sufficient to provoke PNS autoimmunity in athymic recipients, resulting in increased frequency of autoreactive $\mathrm{P} 0$-specific $\mathrm{CD} 4^{+} \mathrm{T}$ cells in the periphery (13). Together, these findings implicate defective central tolerance toward $\mathrm{P} 0$ in driving aberrant immune responses against the PNS (Figure $1 \mathrm{~A})$. Interestingly, heterozygous P0-knockout mice develop a peripheral neuropathy resembling CIDP (20). One possible explanation for this is the decreased $\mathrm{P} 0$ expression in the thymi of the heterozygous P0-knockout mice may 

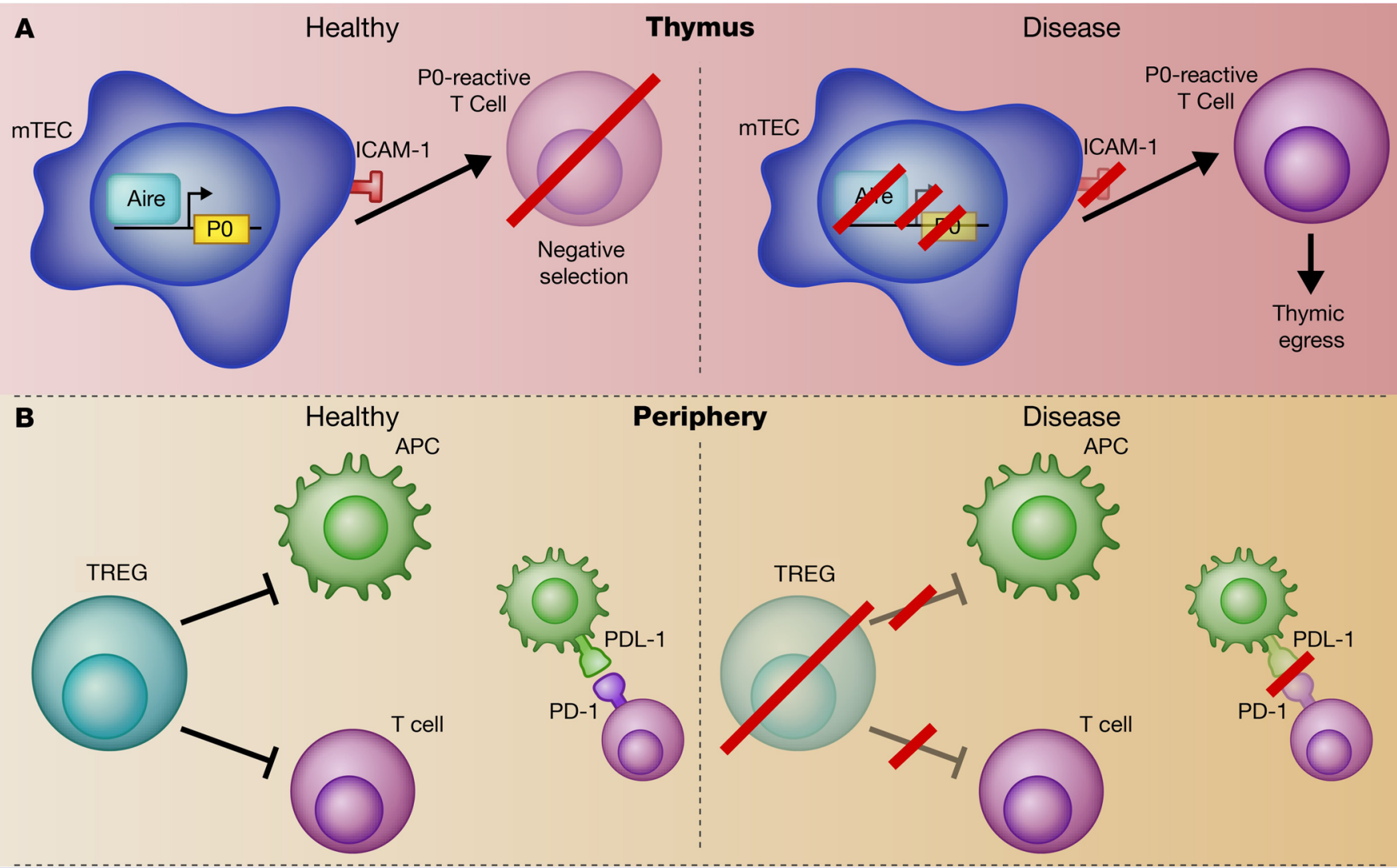

C

Production of Inflammatory

Peripheral Nerves Cytokines by Autoreactive T cells

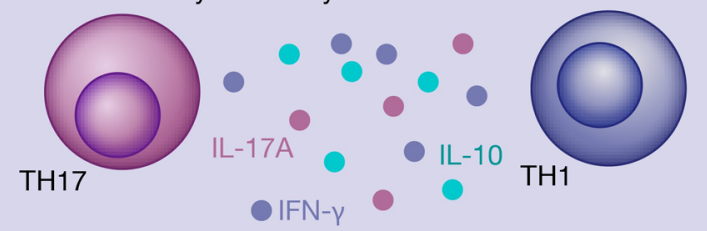

Myelin stripping by

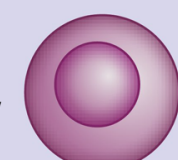
B cell chemotaxis macrophages
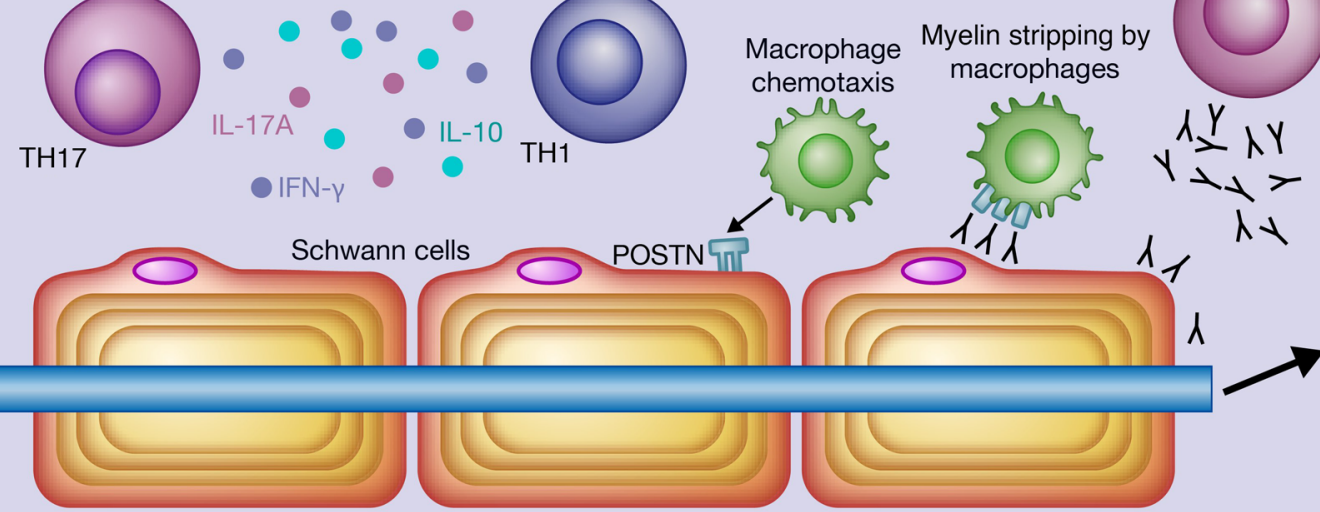

Figure 1. CIDP pathogenesis. Schematic representation of tolerance mechanisms important in preventing PNS autoimmunity and pathogenic mechanisms that lead to CIDP. (A) In a physiologically healthy state, PO-reactive T cells undergo negative selection. In the thymus, AIRE controls expression of tissue-specific antigens, such as PO, and recognition of these antigens by developing T cells leads to their negative selection. In CIDP, loss of negative selection by decreased AIRE or ICAM1 expression in the thymus results in escape of autoreactive T cells into the periphery. (B) Peripheral tolerance mechanisms, including immunosuppressive Treg activity and PD-1/PDL-1 ligation, have also been implicated in preventing PNS autoimmunity. (C) T cell infiltration past the blood-nerve barrier causes an inflammatory environment in the peripheral nerves. CD4+ $T$ cells secrete various cytokines (IFN- $\gamma$, IL-17) that are involved in the immunopathology of CIDP. Macrophage chemotaxis is promoted by POSTN expression by nerve-resident Schwann cells. Autoreactive antibodies produced by B cells are recognized by the Fc receptors on macrophages, which then cause nerve damage by demyelination. Autoantibodies targeted to paranodal proteins (e.g., NF-155) and nodal proteins (e.g., NF-140) are pathogenic in a distinct subset of atypical CIDP. Autoantibodies targeted to peripheral myelin proteins are also found in more generalized forms of CIDP. Illustrated by Rachel Davidowitz. 
result in a defective negative selection of $\mathrm{P} 0$-reactive $\mathrm{T}$ cells and a predisposition to PNS autoimmunity. This underlines the core relevance of $\mathrm{P} 0$ as the dominant antigenic protein in the PNS.

Recent studies have suggested that dominant AIRE mutations are relatively frequent in patients with CIDP (up to 1 in 1000 patients) (21). It remains to be determined, however, whether such mutations, or loss-of-function polymorphisms in AIRE or ICAM1, are associated with sporadic CIDP development, and a reasonably sized GWAS for CIDP is lacking (22). It is also worth exploring the possibility that polymorphisms in P0 or other peripheral myelin protein antigens may be associated with CIDP development. Notably, in type 1 diabetes mellitus, polymorphisms in the variable number of tandem repeat (VNTR) region of the insulin gene have been associated with disease development $(23,24)$. This is due to decreased expression of insulin in the thymus (and presumably decreased negative selection of insulin-specific $\mathrm{T}$ cells) as the result of particular diabetes-associated insulin VNTR polymorphisms.

In addition to defective central tolerance, loss of peripheral tolerance mechanisms has also been implicated in CIDP pathogenesis (Figure 1B). Tregs are impaired in patients with CIDP $(25,26)$, and neuropathy-prone NOD.B7-2 ${ }^{-/-}$mice exhibited a decrease in the frequency of Tregs and regulatory B cells (Bregs). Adoptive transfer of Tregs, but not Bregs, ameliorated progression of spontaneous autoimmune polyneuropathy (SAP) in NOD.B7-2 $2^{-/-}$recipients (27). In another model, Tregs also ameliorated neuritis induced by transfer of $\mathrm{T}$ cells from NOD.ICAM1 ${ }^{\mathrm{tm} 1 \mathrm{Jcgr}}$ mice (28). Interestingly, the absence of B7-2 costimulatory molecules on DCs may prevent peripheral tolerance induction to P0 antigen and may occur through decreased Treg generation (29). Together, these findings suggest that Tregs normally play an important role in preventing PNS autoimmunity.

Another mechanism of peripheral tolerance is the programmed death ligand signaling axis (PD-1/ PDL-1) that inhibits T cell responses in the periphery (15). NOD.H-2 ${ }^{\mathrm{b}} \cdot \mathrm{PD}-1^{-1-}$ mice, which lack PD-1 in the context of antidiabetogenic H-2 haplotype, develop autoimmune PNS inflammation, highlighting the importance of PD-1 in controlling the autoreactive T cell response in the context of $\mathrm{H}-2^{\mathrm{b}} \mathrm{MHC}$ allele. Interestingly, polyneuropathies have been reported as an autoimmune side effect, or immune-related adverse event (IRAE), of anti-PD-1 antibody therapies in cancer $(30,31)$. This suggests that blocking PD-1 in humans may also break immune tolerance toward the PNS. Given the increasing indications for such checkpoint inhibition in cancer, the numbers of patients with IRAEs resembling inflammatory neuropathy are likely to increase. The available case reports indicate that checkpoint inhibitor-associated autoimmune neuritis may feature an increase in cell numbers in the cerebrospinal fluid (CSF) (in addition to the known increase of protein in this compartment) and may respond better to glucocorticoids than IVIg (32). Whether inflammatory checkpoint inhibition-associated neuropathies will mechanistically differ from spontaneous disease remains to be determined.

\section{Immune effectors in CIDP}

T cells. CD4+ Th cells are a prominent immune cell type found in the infiltrates of CIDP nerve biopsies (33). Their important role in disease pathogenesis has been emphasized using CIDP mouse models. Adoptive transfer of $\mathrm{CD}^{+} \mathrm{T}$ cells purified from neuropathic NOD.Aire ${ }^{\mathrm{GW} /+}$ and NOD.B7 $-2^{-/-}$mice is sufficient to transfer disease to immunodeficient recipients. Additionally, a $\mathrm{P} 0$-specific $\mathrm{CD} 4^{+} \mathrm{T}$ cell receptor transgenic (P0T) mouse was generated from PNS-derived CD4 ${ }^{+} \mathrm{T}$ cells from NOD.B7-2 $2^{-/}$mice. When crossed onto the $\mathrm{T}$ and $\mathrm{B}$ cell-deficient recombination activating gene (RAG) knockout background, P0T mice spontaneously develop neuropathy associated with immune cell infiltration into peripheral nerves $(12,34)$.

$\mathrm{CD} 4{ }^{+}$Th cells can differentiate into multiple subtypes that produce lineage-defining cytokines. Patients with CIDP have a significant increase in both IFN- $\gamma$-producing Th1 and IL-17-producing Th17 cells in the peripheral blood and CSF (25). Studies in mouse models suggest potential roles for both Th1 and Th17 cells in CIDP pathogenesis. IFN- $\gamma$-deficient NOD.Aire $\mathrm{GW}^{\mathrm{H}+}$ and NOD.B7- $2^{-/-}$mice are completely protected from the development of peripheral nerve autoimmunity, suggesting an essential role for IFN- $\gamma$ in disease development $(12,15,35)$. An open question in the field is the contribution of IL-17-producing Th17 cells. In support of the possible contribution of these cells, increased levels of IL-17 were observed during the late stage of chronic experimental autoimmune neuritis in Lewis rats, possibly indicating a role for Th17 cells in the development of chronic forms of autoimmune peripheral neuropathy. Moreover, Th17 cells were also shown to increase in neuropathic NOD.ICAM1 ${ }^{\mathrm{tm} 1 \mathrm{Jcgr}}$ mice (13). Moreover, in an inflammation/ injury model, where peripheral neuropathy was induced in C57BL/6 mice by partial ligation of the sciatic nerve, mice lacking IL-17 had significantly fewer infiltrating $\mathrm{T}$ cells and macrophages and decreased pain 
hypersensitivity (36). Moreover, IL-17 may have direct effects on Schwann cells, through inhibition of myelination and upregulation of MHC-I (37). Further studies are needed to determine whether both IFN- $\gamma$ and IL-17 play critical roles in the immunopathology of CIDP.

Intriguingly, production of the antiinflammatory cytokine IL-10 by CD4 ${ }^{+} \mathrm{T}$ cells has also recently been implicated in the development of autoimmune peripheral neuropathy. IL-10 is upregulated in the peripheral nerves of patients with $\operatorname{CIDP}(38,39)$. Notably, IL-10 is widely regarded as an immunosuppressive cytokine and has previously been shown to dampen autoimmune diseases, such as multiple sclerosis and rheumatoid arthritis $(40,41)$. Thus, it was surprising that IL-10-deficient NOD.Aire ${ }^{\mathrm{GW} /+}$ mice exhibit a significant delay in the onset of SAP (38), suggesting a potential proinflammatory role for IL-10 in the development of autoimmune peripheral neuropathy. In this experimental setting, IL-10 deficiency in CD4 ${ }^{+}$ $\mathrm{T}$ cells prevented adoptive transfer of neuropathy, suggesting that IL-10 production by $\mathrm{CD} 4^{+} \mathrm{Th}$ cells is required for disease transfer. Interestingly, IL-10 promotes the autoimmune response by inducing $\mathrm{CD} 4^{+} \mathrm{T}$ cell egress from the lymph nodes into the peripheral nerves in a sphingosine-1-phosphate receptor 1-dependent (S1PR1-dependent) manner (38). Additional evidence supporting a proinflammatory role for IL-10 stems from a transgenic mouse line on the C57BL/ 6 background. In these mice, IL-10 overexpression, via the vitelliform macular dystrophy 2 (VMD-2) promoter, induces spontaneous autoimmune peripheral neuropathy (42). Interestingly, IL-10 expression induces an influx of macrophages, suggesting that IL-10 may promote inflammation in peripheral nerves by attracting these innate immune cells. Taken together, these findings suggest an unexpected, proinflammatory role for IL-10 in peripheral nerve autoimmunity.

In addition to $\mathrm{CD}^{+} \mathrm{Th}$ cells, $\mathrm{CD} 8^{+} \mathrm{T}$ cells are also prominent in CIDP nerve biopsies (33). In some reports, $\mathrm{CD}^{+} \mathrm{T}$ cells even outnumbered $\mathrm{CD} 4^{+} \mathrm{T}$ cells in the lesions of patients with $\mathrm{CIDP}(43,44)$. Cytotoxic $\mathrm{CD}^{+} \mathrm{T}$ cells have been implicated in the antigen-driven MHC class I-restricted attack against PNS components, as shown in CIDP patient blood and corresponding nerve biopsies (45). Moreover, peripheral blood $\mathrm{CD}^{+} \mathrm{T}$ cells from patients with CIDP have extensive oligoclonal expansion compared with CD4 ${ }^{+}$ $\mathrm{T}$ cells. More recently, we found that CSF composition of patients with CIDP is disease specific, with a significant increase in cytotoxic cell types NKT cells and CD8 ${ }^{+} \mathrm{T}$ cells $(45,46)$. In mouse CIDP models, the role of $\mathrm{CD}^{+} \mathrm{T}$ cells requires further clarification. The contribution of $\mathrm{CD} 8^{+} \mathrm{T}$ cells to the spontaneous demyelination of the PNS has been suggested in an animal model of a more acute type of neuropathy (47). However, in other mouse models, purified $\mathrm{CD} 8^{+} \mathrm{T}$ cells are unable to transfer disease on their own $(12,48)$. Thus, the pathogenic role of $\mathrm{CD}^{+} \mathrm{T}$ cells remains unclear.

$B$ cells and antibodies. The role of B cells and antibodies in CIDP remains to be determined. In support of a role for autoantibody-producing B cells, a subtype of rapidly progressive CIDP associated with tremor and lack of IVIg response has been attributed to pathogenic IgG4 autoantibodies against paranodal proteins contactin-1 (CNTN1), CASPR1, and neurofascin 155 (NF-155) (refs. 49, 50 and Figure 1C). In addition, autoantibodies against NF-155 isolated from human patient samples were not reactive in NF-155-deficient mice, indicating specific target reactivity to paranodal proteins (51). These paranodal proteins are important for the adhesion of myelin sheath borders to axons at the paranodes, and autoantibodies against CNTN1 and NF-155 have been demonstrated to disrupt this function $(52,53)$. Additionally, IgG4 or IgG3 autoantibodies against neuronal isoforms of NF-140 and NF-186, located at the nodes of Ranvier, have also been described in 5 patients with CIDP, with a severe phenotype associated with conduction block. None of the patients had tremor, and three of the five patients responded to IVIg and glucocorticoid treatment, which distinguishes these patients from those with IgG4 antibodies against paranodal proteins (54). Thus, antibodies against paranodal and nodal proteins may represent a subset of patients with atypical CIDP with clinically distinct features.

In typical forms of CIDP, increased frequencies of autoantibodies that react against peripheral nerve myelin proteins have been reported in sera of patients with CIDP (55). Western blot-based experiments have shown that $25 \%$ of serum samples from patients with CIDP (vs. $5 \%$ of healthy controls) contain antibodies against peripheral nerve proteins, and the most common antibody was directed against a $28-\mathrm{kD}$ protein, suggestive of the P0 protein. In parallel, studies in mouse models also support a role for B cells. First, increased P0-specific autoantibodies have been noted in multiple animal models (12, 34). Second, neuropathy development is attenuated in B cell-deficient NOD.B7-2 $2^{-/}$mice, indicating a contribution of pathogenic B cells to disease (56). Additionally, P0-specific plasmablasts and B cells are increased in CIDP mouse models, and targeting these cell types with anti-CD19 attenuated disease severity and decreased the level of autoreactive IgM and IgG antibodies in the serum (57). Finally, the efficacy of plasmapheresis as a therapy for CIDP suggests a role for a humoral immune component. 
Arguing against an important role for autoantibodies, however, is the observation that autoantibody responses against a particular myelin protein occur in only a small minority of patients with typical CIDP (55). Moreover, while IgG4 antibodies against paranodal and nodal proteins have been shown to be pathogenic, IgG4-associated CIDP accounts for only approximately $10 \%$ of CIDP cases. Thus, the importance of autoantibodies in the majority of patients with CIDP remains to be determined. In the NOD.Aire ${ }^{\mathrm{GW} /+}$ mouse model, genetic B cell deficiency did not prevent autoimmune neuropathy development (38). Why B cell deficiency is protective in the NOD.B7-2 $2^{-/-}$model but not in the NOD.Aire $\mathrm{GW}^{/+}$ is unclear. B7-2 deficiency has been reported to result in compensatory upregulation of B7-1 (48). Thus, it is possible that the B7-1 upregulation on B cells promotes autoimmunity in NOD.B7-2-/- mice. In support of this hypothesis, pathogenic $\mathrm{T}$ cell activation in inflammatory insulitis has been attributed to dysregulated expression of B7-1 and -2 on B cells (58). Interestingly, rituximab (anti-CD20 antibody) has been used with variable efficacy in CIDP but appears to have increased efficacy among patients with CIDP with IgG4 anti-CNTN1/NF155 nodal proteins (59). Together, these findings suggest variable roles for B cells and/or autoantibodies among CIDP subtypes.

Macrophages. In CIDP nerve biopsies, macrophages are the predominant cell type within the immune infiltrate. Multiple proinflammatory functions have been attributed to macrophages (60). Electron microscopy of CIDP nerve biopsies has clearly depicted macrophages insinuating the myelin folds of healthy-appearing Schwann cells, suggesting that macrophages attack intact myelin from peripheral nerves. This attack could be secondary to opsonization of myelin, as antibodies on myelin targets may bind $\mathrm{Fc}$ receptors on macrophages, thereby directing macrophages to the nerves. Additionally, macrophages may phagocytose myelin sheaths that are left behind after Wallerian degeneration (60). Consistent with a pathogenic role for macrophages, depletion of phagocytic cells in NOD. Aire ${ }^{\mathrm{GW} /+}$ mice protected mice from neuropathy development (61). In addition, macrophages participate in the autoimmune response through secretion of proinflammatory cytokines, direct destruction of target cells, and by presentation of antigens.

At the same time, macrophages have well-described roles in nerve regeneration. After injury, macrophages clear debris and regulate Schwann cell remyelination, in part through secretion of Gas6 (62). Thus, macrophages have seemingly dichotomous roles, both promoting demyelination and Schwann cell remyelination. These conflicting roles may reflect heterogeneity within the macrophage population, and further studies will be required to understand the role of particular macrophage subtypes in the nerve microenvironment and require approaches that enable dissection of these cells at unprecedented resolution (see below).

\section{Nerve-resident cells in inflammation}

PNS antigens, including Schwann cell-expressed myelin antigens, are targeted by the autoimmune responses in CIDP. Notably, it is now clear that PNS-intrinsic cells are not only the target of autoimmunity, but also play a major role in disease outcome. An early clue came from the observation that CIDP nerve biopsies were characterized by "onion bulbs" (2). These structures are supernumerary Schwann cell processes and are indicative of repetitive segmental degeneration and regeneration of myelin. The PNS has the potential to extensively regenerate after damage in a process supported by dedifferentiated Schwann cells $(63,64)$. Recent work has uncovered multiple mechanisms that control Schwann cell reprogramming and subsequent nerve regeneration (65-67). Together, these findings suggest that nerve regeneration, in addition to immune destruction, could also play an important role in CIDP disease outcome.

In addition to nerve regeneration, Schwann cells may also control immune cell chemotaxis into the PNS. With the onset of immune cell infiltration, Schwann cells in NOD.Aire ${ }^{\mathrm{GW} /+}$ mice and patients with CIDP upregulate expression of the extracellular matrix protein periostin (POSTN) (61). POSTN expression promotes autoimmune destruction by increasing chemotaxis of pathogenic macrophages into the affected nerve, as Postn deficiency in NOD.Aire ${ }^{\mathrm{GW} /+}$ mice slowed disease onset and decreased macrophage infiltration. Furthermore, a study in neuropathic NOD.B7-2 ${ }^{-/-}$mice highlights a role for fibronectin-connecting segment 1 (FCNS1) on microvascular endothelial cells. FCNS1 is an alternative binding partner of integrin $\alpha_{4}$ (CD49d) on proinflammatory leukocytes, and blocking FCNS1 in this mouse model inhibits development of PNS autoimmunity. In nerve biopsies of patients with CIDP, FCNS1 is upregulated in microvessels, and anti-FCNS1 antibodies inhibit trafficking across the human BNB in vitro (68). These studies, thus, demonstrate the importance of understanding how PNS-intrinsic cells are altered with PNS autoimmunity and how they participate in the inflammatory process. 


\section{Emerging approaches to understanding human CIDP}

Although mouse models display multiple features of human CIDP, some interspecies differences have been difficult to reconcile. For instance, in mouse models, $\mathrm{P} 0$ has been identified as a dominant antigen targeted by autoimmunity. In human CIDP, however, multiple neural antigens have been identified as targets of autoimmunity. This is likely because CIDP is not a single entity, but rather composed of multiple variants with complex pathogenic mechanisms. As such, not one mouse model can mimic the heterogeneity of the human disease. Thus, studying patient samples is critical for further deciphering disease pathogenesis. Multiple approaches have been used to study samples from patients with CIDP. Histology (33, 45, 69 ) and electron microscopy $(70,71)$ on nerve biopsies from patients with CIDP has provided important information about CIDP pathogenesis. A deeper understanding of molecular mechanisms was gained by FACS analysis of patient blood (72) and CSF $(46,72)$. Notably, transcriptomic technologies are beginning to reveal a much higher resolution view of the cellular composition of the PNS in homeostasis and CIDP disease development.

Transcriptomic approaches. Initial transcriptomic studies used microarray-based gene expression profiling on mouse and rat sciatic nerves at different embryonic stages to map the developmental stages of Schwann cells and their precursors (73). In the human disease setting, sural nerve biopsies from patients with CIDP showed disease-related transcriptional patterns $(74,75)$. These studies identified induction of genes associated with pain sensation, remyelination, and immune responses in CIDP. Another study applied microarrays on skin biopsies of patients with CIDP and detected similar differentially expressed genes, all reported to be involved in inflammatory or immune processes (76). Microarray has also been applied to study the response of patients with CIDP to IVIg therapy $(76,77)$, showing potential of this approach to serve as a diagnostic and potentially even prognostic tool. However, microarray technology is limited by its inability to identify unknown genes and the high amounts of input material that are required.

RNA-seq quantifies gene expression by sequencing RNA after reverse transcription into cDNA. RNAseq requires less input material than other approaches and allows identification of new gene transcripts (Figure 2A). This technique has identified not only transcriptional changes of sciatic nerve cells that are associated with tissue regeneration and nerve injury (76-78) but also the pathophysiology of hereditary neuropathies (79) and regulation of neuropathic pain $(79,80)$. In addition, neurons, dorsal root ganglia (DRG), and the BNB have been subjected to RNA-seq $(81,82)$, albeit not in the specific setting of CIDP and its models. In addition, microarray and bulk RNA-seq do not identify cell-specific transcriptomes, because in the combined bulk tissue, the cells of interest are mixed with other cell types. The recent advent of single-cell transcriptomic techniques promises to enable a considerably more detailed look at many tissues but has not yet been used in CIDP.

Single-cell RNA-seq (scRNA-seq) enables the discovery of new cell types by defining the cellular composition in an unbiased way. Although original techniques require manually obtained single cells, more recent microfluidic technologies offer semiautomated single-cell processing and have gained considerable popularity over the last years (83-86). In microfluidics-based scRNA-seq techniques, mRNA from individual cells is reverse transcribed and labeled with cell-specific barcodes immediately after cell lysis. To achieve this, single cells are brought together in a high-throughput fashion with oligonucleotide-labeled gel beads, lysis buffer, and reverse transcription mix in individual water-in-oil microdroplets (refs. 83, 84, 86 and Figure 2B). The oligonucleotides on the gel beads contain unique molecular identifier (UMI) sequences that allow correction for biases in cDNA amplification (87). An additional stretch of oligonucleotide sequence allows identification of single cells after sequencing $(83,84)$. This barcoding allows manual processing of cDNA from many cells combined and thus is highly cost-effective. After the reverse transcription, which takes place within the water-in-oil microdroplet, cDNAs are pooled for amplification, library preparation, and sequencing.

Single-cell transcriptomics allow redefining of the cellular complexity within any tissue of interest. For example, DRG that house the sensory neuronal cell bodies extending into the PNS have been characterized using single-cell transcriptomics under both steady-state conditions and after injury (88-90). More recently, scRNA-seq characterization of the PNS was reported; it was used to analyze traumatically injured rodent sciatic nerves after enzymatic digestion, followed by droplet-based scRNA-seq $(88,91)$. This analysis identified multiple cell types, including mesenchymal cells, Schwann cells, endothelial cells, and leukocytes. The study focused on the regenerative capacity of mesenchymal cells and identified cells that transcriptionally resemble mesenchymal progenitor cells that migrate to damaged tissue and contribute to repair (91). 

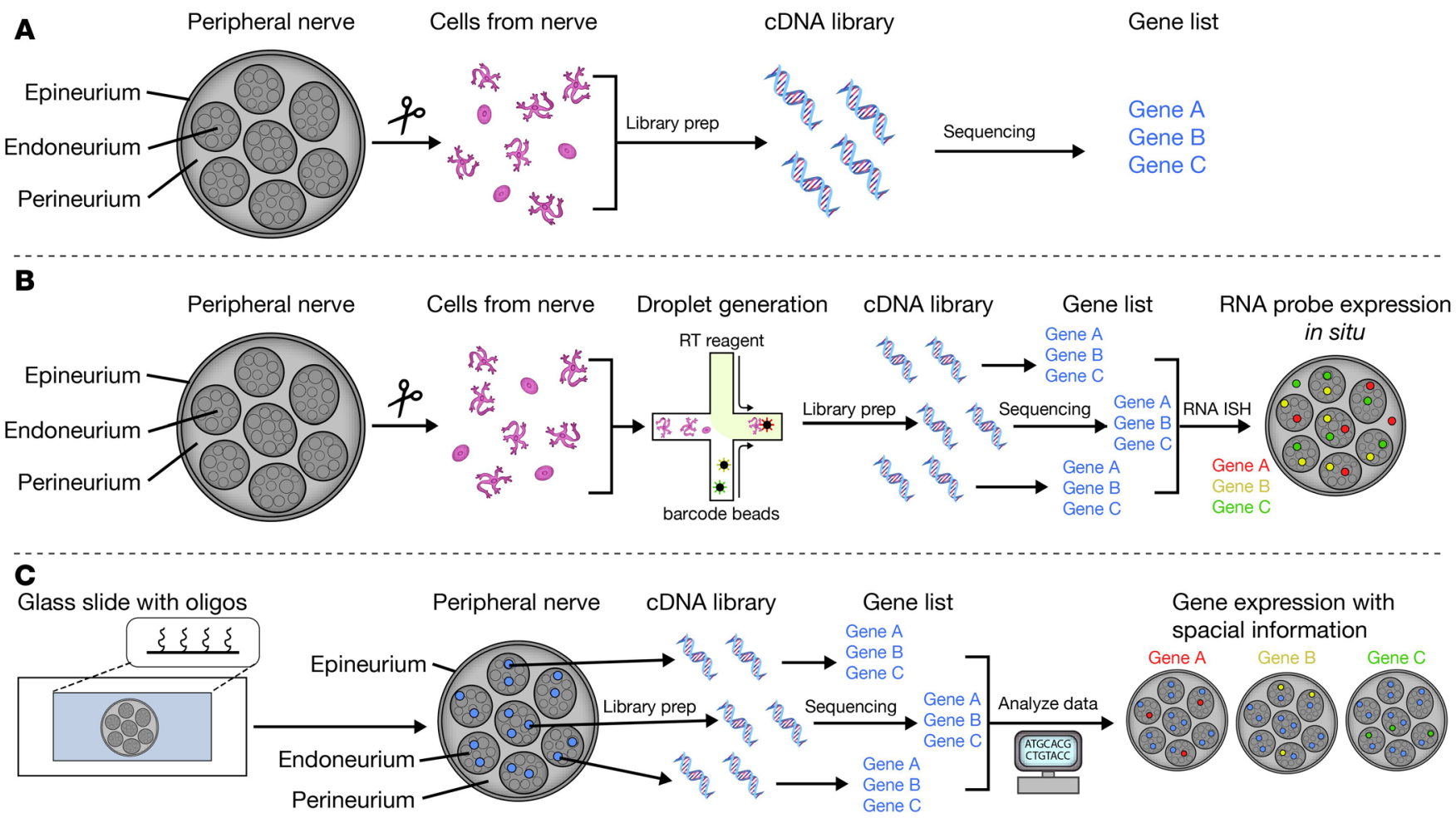

Figure 2. Overview of recently developed RNA-seq techniques. (A) Whole genome bulk RNA-seq involves the digestion of tissue and analysis of all transcripts pooled together. This analysis is only tissue or cell specific when performed on presorted, fluorescently labeled cells. (B) scRNA-seq combined with (multiplex) RNA in situ hybridization. scRNA-seq still involves tissue digestion, but transcriptome analysis reaches a single-cell level. Microfluidics techniques are used to bring cells together with oligo-labeled beads and lysis buffer in droplets to allow the identification of all single cells after sequencing at the bulk level. Spatial information from marker genes can be acquired by multiplexed RNA in situ hybridization techniques of known RNA probes or computational comparison with known gene expression atlases. (C) Whole tissue sections are permeabilized on top of glass slides bound with oligonucleotides encoding spatial information. Barcoded transcriptomes are processed for genome-wide RNA-seq. With an optimized resolution of $2 \mu \mathrm{m}$, this new technique makes it possible to detect the single-cell transcriptome without losing the tissue spatial orientation. Illustrated by Rachel Davidowitz.

However, a detailed cellular response of PNS parenchymal cells to local autoimmune reactions has not been described $(92,93)$.

We recently applied a similar dissociation protocol to mouse sciatic nerves from healthy mice and mice developing an autoimmune neuropathy and analyzed these cells via scRNA-seq (our unpublished observations) (Figure 2B). We focused on immune cell populations within the peripheral nerve and their interactions with the parenchymal cells using the NOD.ICAM1 ${ }^{\text {mIIsgr }}$ mouse model (see above) (Wolbert et al., personal communication). In this model, T and B cells as well as plasmacytoid DCs were expanded in the PNS before the appearance of clinical symptoms and altered the local cell-cell communication circuitry. Surprisingly, two transcriptionally distinct populations of homeostatic endoneurial macrophages were identified with potentially distinct function. This technique may be translatable to human sciatic nerves to aid in understanding disease pathogenesis and diagnosing patients with autoimmune and/or inflammatory neuropathies, such as CIDP.

In a heterogeneous disease such as CIDP, prognostic and diagnostic markers are often challenging to identify (e.g., see ref. 94). scRNA-seq has been used to identify new biomarkers and therapeutic targets for complex diseases. One study constructed network models of disease-associated cell types previously identified in mouse models and human patients with rheumatoid arthritis, thereby prioritizing cells types and genes for diagnostics and therapeutics in human disease (95). Another study used scRNA-seq to identify drug target pathway activation in a patient with renal carcinoma (96), showing the potential of scRNA-seq for clinical utility and design of personalized medicine. Although databases are not yet available for the PNS in human CIDP, these previous studies may pave the way to using this technique to improve diagnostic and therapeutic approaches in CIDP.

Future possibilities - spatial transcriptomics. Despite its analytical power, scRNA-seq requires dissociation of the tissue of interest and, therefore, results in a loss of spatial information. The technical possibility of 
combining genome-wide transcriptional analysis with spatial information has recently been established. One approach is to sequentially use scRNA-seq to identify marker genes and then perform multiplex RNA in situ hybridization to visualize and, thus, localize the previously identified transcripts (e.g., refs. 97-99; Figure 2B). Single-cell mRNA profiles have also been compared with positional gene expression profiles derived from a gene expression atlas (97). Another study generated in parallel a spatial database of landmark gene expression. The spatial origin of a cell could then be predicted based on the expression of verified landmark genes $(97,98,100)$. Although these methods provide important information, they have limitations in the number of genes that can be simultaneously analyzed and are not fully unbiased.

More recently, RNA-seq data has been obtained from single cells (or a few cells), while maintaining the spatial information within the tissue $(101,102)$, and has been named spatial transcriptomics. Specifically, histological sections are placed onto glass slides that are labeled with unique spatially barcoded capture probes, with spatial information encoded within the barcode. Spatially barcoded genome-wide transcriptomes are subsequently obtained using protocols similar to scRNA-seq (Figure 2C). Although the currently available spatial resolution of $100 \mu \mathrm{m}$ covers only 5-100 cells at once, high-density spatial transcriptomic arrays have recently been introduced that achieve $2-\mu \mathrm{m}$ resolution (99). Several spatial transcriptomics studies have been done on CNS tissue $(103,104)$. Obvious issues left to address in the PNS include deciphering differences between proximal and distal nerve segments, the ensheathing of sensory verses motor axons by Schwann cells, and also changes in tissue organization and pathophysiology during disease (105). Combining single-cell transcriptomics with anatomical information will aid in the understanding of PNS basic cell biology and pathophysiology in rodents and humans.

\section{Conclusions}

In this Review, we have described how diverse components of the immune system drive and control inflammatory processes in the PNS and how animal models have facilitated a deep mechanistic understanding of chronic PNS autoimmunity. New methods are beginning to enable a truly "holistic" view of cellular alterations and interactions of parenchymal cells and immune cells in nervous tissue in situ. Such technical developments, combined with established animal models, will likely allow the identification of marker genes of previously underappreciated cell subsets and also provide more information on cell differentiation states, lineage development, and interactions in health and autoimmune peripheral neuropathies for the ultimate benefit of affected patients.

\section{Acknowledgments}

GMZH was supported in part by the Deutsche Forschungsgemeinschaft (ME4050/4-1, ME4050/8-1), by the Innovative Medical Research program of the University of Münster, by the Ministerium für Innovation, Wissenschaft und Forschung of the state "Nordrhein-Westfalen," and by the Grant for Multiple Sclerosis Innovation from Merck.

MAS was supported by the NIH National Institute of Neurological Disorders and Stroke, the National Organization for Rare Diseases, and the Parker Institute for Cancer Immunotherapy.

Address correspondence to: Maureen Su, 615 Charles E. Young Drive South, Los Angeles, California 90095, USA. Phone: 310.825.2130; Email: masu@mednet.ucla.edu. Or to: Gerd Meyer zu Horste, AlbertSchweitzer-Campus 1A, 48149 Münster, Germany. Phone: 49.251.8346810; Email: gerd.mzh@uni-muenster.de.

1. Dalakas MC. Advances in the diagnosis, pathogenesis and treatment of CIDP. Nat Rev Neurol. 2011;7(9):507-517.

2. Dyck PJ, Lais AC, Ohta M, Bastron JA, Okazaki H, Groover RV. Chronic inflammatory polyradiculoneuropathy. Mayo Clin Proc. 1975;50(11):621-637.

3. McCombe PA, Pollard JD, McLeod JG. Chronic inflammatory demyelinating polyradiculoneuropathy. A clinical and electrophysiological study of 92 cases. Brain. 1987;110(Pt 6):1617-1630.

4. Hughes R, Cornblath D, Jacobs B. International Guilain-Barré syndrome outcome study (IGOS): a prospective study on clinical and biological predictors of disease course and outcome. Paper presented at: Meeting of the Peripheral Nerve Society; June 29-July 3, 2013; Saint-Malo, France. http://air.unimi.it/handle/2434/253143. Accessed January 24, 2020.

5. Jacobs BC, et al. International Guillain-Barré Syndrome Outcome Study: protocol of a prospective observational cohort study on clinical and biological predictors of disease course and outcome in Guillain-Barré syndrome. J Peripher Nerv Syst. 2017;22(2):68-76 
6. Bunschoten C, Eftimov F, van der Pol WL, Jacobs BC, ICOS Consortium. International chronic inflammatory demyelinating polyneuropathy outcome study (ICOS): Protocol of a prospective observational cohort study on clinical and biological predictors of disease course and outcome. J Peripher Nerv Syst. 2019;24(1):34-38.

7. Gorson KC, et al. Chronic inflammatory demyelinating polyneuropathy disease activity status: recommendations for clinical research standards and use in clinical practice. J Peripher Nerv Syst. 2010;15(4):326-333.

8. Soliven B. Animal models of autoimmune neuropathy. ILAR J. 2014;54(3):282-290.

9. Schafflick D, Kieseier BC, Wiendl H, Meyer Zu Horste G. Novel pathomechanisms in inflammatory neuropathies. J Neuroinflammation. 2017;14(1):232.

10. Bour-Jordan H, Thompson HL, Giampaolo JR, Davini D, Rosenthal W, Bluestone JA. Distinct genetic control of autoimmune neuropathy and diabetes in the non-obese diabetic background. J Autoimmun. 2013;45:58-67.

11. Su MA et al. Mechanisms of an autoimmunity syndrome in mice caused by a dominant mutation in Aire. J Clin Invest. 2008;118(5):1712-1726.

12. Su MA, et al. Defective autoimmune regulator-dependent central tolerance to myelin protein zero is linked to autoimmune peripheral neuropathy. J Immunol. 2012;188(10):4906-4912.

13. Meyer zu Horste G, et al. Thymic epithelium determines a spontaneous chronic neuritis in Icam1(tm1Jcgr)NOD mice. J Immunol. 2014;193(6):2678-2690.

14. Salomon B, et al. Development of spontaneous autoimmune peripheral polyneuropathy in B7-2-deficient NOD mice. $J$ Exp Med. 2001;194(5):677-684

15. Yoshida T, Jiang F, Honjo T, Okazaki T. PD-1 deficiency reveals various tissue-specific autoimmunity by H-2b and dose-dependent requirement of H-2g7 for diabetes in NOD mice. Proc Natl Acad Sci USA. 2008;105(9):3533-3538.

16. Gold R, Archelos JJ, Hartung HP. Mechanisms of immune regulation in the peripheral nervous system. Brain Pathol. 1999;9(2):343-360.

17. Kieseier BC, Lehmann HC, Meyer Zu Hörste G. Autoimmune diseases of the peripheral nervous system. Autoimmun Rev. 2012;11(3):191-195.

18. Dalakas MC. Pathogenesis of immune-mediated neuropathies. Biochim Biophys Acta. 2015;1852(4):658-666.

19. Valenzise $\mathrm{M}$, et al. Chronic inflammatory demyelinating polyneuropathy as a possible novel component of autoimmune poly-endocrine-candidiasis-ectodermal dystrophy. Eur J Pediatr. 2009;168(2):237-240.

20. Shy ME, et al. Heterozygous P0 knockout mice develop a peripheral neuropathy that resembles chronic inflammatory demyelinating polyneuropathy (CIDP). J Neuropathol Exp Neurol. 1997;56(7):811-821.

21. Oftedal BE, et al. Dominant mutations in the autoimmune regulator AIRE are associated with common organ-specific autoimmune diseases. Immunity. 2015;42(6):1185-1196.

22. Blum S, McCombe PA. Genetics of Guillain-Barré syndrome (GBS) and chronic inflammatory demyelinating polyradiculoneuropathy (CIDP): current knowledge and future directions. J Peripher Nerv Syst. 2014;19(2):88-103.

23. Lucassen AM, et al. Susceptibility to insulin dependent diabetes mellitus maps to a $4.1 \mathrm{~kb}$ segment of DNA spanning the insulin gene and associated VNTR. Nat Genet. 1993;4(3):305-310.

24. Vafiadis P, et al. Insulin expression in human thymus is modulated by INS VNTR alleles at the IDDM2 locus. Nat Genet. 1997;15(3):289-292.

25. Chi LJ, Xu WH, Zhang ZW, Huang HT, Zhang LM, Zhou J. Distribution of Th17 cells and Th1 cells in peripheral blood and cerebrospinal fluid in chronic inflammatory demyelinating polyradiculoneuropathy. J Peripher Nerv Syst. 2010;15(4):345-356.

26. Chi LJ, Wang HB, Wang WZ. Impairment of circulating CD4+CD25+ regulatory T cells in patients with chronic inflammatory demyelinating polyradiculoneuropathy. J Peripher Nerv Syst. 2008;13(1):54-63.

27. Quan S, Sheng JR, Abraham PM, Soliven B. Regulatory T and B lymphocytes in a spontaneous autoimmune polyneuropathy. Clin Exp Immunol. 2016;184(1):50-61.

28. Meyer zu Hörste G, et al. FoxP3+ regulatory T cells determine disease severity in rodent models of inflammatory neuropathies. PLoS ONE. 2014;9(10):e108756.

29. Quan S, Kim HJ, Dukala D, Sheng JR, Soliven B. Impaired dendritic cell function in a spontaneous autoimmune polyneuropathy. J Immunol. 2015;194(9):4175-4184.

30. Mancone S, et al. Severe neurologic complications of immune checkpoint inhibitors: a single-center review. J Neurol. 2018;265(7):1636-1642.

31. Tanaka R, et al. Nivolumab-induced chronic inflammatory demyelinating polyradiculoneuropathy mimicking rapid-onset Guillain-Barré syndrome: a case report. Jpn J Clin Oncol. 2016;46(9):875-878

32. Supakornnumporn S, Katirji B. Guillain-Barré Syndrome Triggered by Immune Checkpoint Inhibitors: A Case Report and Literature Review. J Clin Neuromuscul Dis. 2017;19(2):80-83.

33. Cornblath DR, Griffin DE, Welch D, Griffin JW, McArthur JC. Quantitative analysis of endoneurial T-cells in human sural nerve biopsies. J Neuroimmunol. 1990;26(2):113-118.

34. Louvet $\mathrm{C}$, et al. A novel myelin P0-specific $\mathrm{T}$ cell receptor transgenic mouse develops a fulminant autoimmune peripheral neuropathy. J Exp Med. 2009;206(3):507-514.

35. Bour-Jordan H, Thompson HL, Bluestone JA. Distinct effector mechanisms in the development of autoimmune neuropathy versus diabetes in nonobese diabetic mice. J Immunol. 2005;175(9):5649-5655.

36. Kim CF, Moalem-Taylor G. Interleukin-17 contributes to neuroinflammation and neuropathic pain following peripheral nerve injury in mice. J Pain. 2011;12(3):370-383.

37. Stettner M, et al. Interleukin-17 impedes Schwann cell-mediated myelination. J Neuroinflammation. 2014;11:63.

38. Smith CJ, Allard DE, Wang Y, Howard JF, Montgomery SA, Su MA. IL-10 Paradoxically promotes autoimmune neuropathy through S1PR1-dependent CD4+ T cell migration. J Immunol. 2018;200(5):1580-1592.

39. Sanvito L, Makowska A, Gregson N, Nemni R, Hughes RA. Circulating subsets and CD4(+)CD25(+) regulatory T cell function in chronic inflammatory demyelinating polyradiculoneuropathy. Autoimmunity. 2009;42(8):667-677

40. Guo B. IL-10 modulates Th17 pathogenicity during autoimmune diseases. J Clin Cell Immunol. 2016;7(2):400.

41. Iyer SS, Cheng G. Role of interleukin 10 transcriptional regulation in inflammation and autoimmune disease. Crit Rev Immunol. 
2012;32(1):23-63

42. Dace DS, Khan AA, Stark JL, Kelly J, Cross AH, Apte RS. Interleukin-10 overexpression promotes Fas-ligand-dependent chronic macrophage-mediated demyelinating polyneuropathy. PLOS ONE. 2009;4(9):e7121

43. Zhang Z, Zhang ZY, Fauser U, Schluesener HJ. Distribution of Foxp3(+) T-regulatory cells in experimental autoimmune neuritis rats. Exp Neurol. 2009;216(1):75-82.

44. Matsumuro K, Izumo S, Umehara F, Osame M. Chronic inflammatory demyelinating polyneuropathy: histological and immunopathological studies on biopsied sural nerves. J Neurol Sci. 1994;127(2):170-178.

45. Schneider-Hohendorf T, Schwab N, Uçeyler N, Göbel K, Sommer C, Wiendl H. CD8+ T-cell immunity in chronic inflammatory demyelinating polyradiculoneuropathy. Neurology. 2012;78(6):402-408.

46. Heming M, et al. Immune cell profiling of the cerebrospinal fluid provides pathogenetic insights into inflammatory neuropathies. Front Immunol. 2019;10:515.

47. Yang M, et al. Evidence from human and animal studies: pathological roles of $\mathrm{CD} 8(+) \mathrm{T}$ cells in autoimmune peripheral neuropathies. Front Immunol. 2015;6:532.

48. Salomon B, et al. Development of spontaneous autoimmune peripheral polyneuropathy in B7-2-deficient NOD mice. J Exp Med. 2001;194(5):677-684.

49. Querol L, Illa I. Paranodal and other autoantibodies in chronic inflammatory neuropathies. Curr Opin Neurol. 2015;28(5):474-479.

50. Querol L, et al. Neurofascin IgG4 antibodies in CIDP associate with disabling tremor and poor response to IVIg. Neurology. 2014;82(10):879-886.

51. Vallat JM, et al. Paranodal lesions in chronic inflammatory demyelinating polyneuropathy associated with anti-Neurofascin 155 antibodies. Neuromuscul Disord. 2017;27(3):290-293.

52. Labasque M, Hivert B, Nogales-Gadea G, Querol L, Illa I, Faivre-Sarrailh C. Specific contactin N-glycans are implicated in neurofascin binding and autoimmune targeting in peripheral neuropathies. J Biol Chem. 2014;289(11):7907-7918.

53. Koike H, et al. Ultrastructural mechanisms of macrophage-induced demyelination in CIDP. Neurology. 2018;91(23):1051-1060.

54. Delmont E, et al. Autoantibodies to nodal isoforms of neurofascin in chronic inflammatory demyelinating polyneuropathy. Brain. 2017;140(7):1851-1858.

55. Allen D, et al. Antibodies to peripheral nerve myelin proteins in chronic inflammatory demyelinating polyradiculoneuropathy. J Peripher Nerv Syst. 2005;10(2):174-180.

56. Quan $\mathrm{H}$, et al. An adaptive immune response driven by mature, antigen-experienced $\mathrm{T}$ and $\mathrm{B}$ cells within the microenvironment of oral squamous cell carcinoma. Int J Cancer. 2016;138(12):2952-2962.

57. Abraham PM, Quan SH, Dukala D, Soliven B. CD19 as a therapeutic target in a spontaneous autoimmune polyneuropathy. Clin Exp Immunol. 2014;175(2):181-191.

58. Hussain S, Delovitch TL. Dysregulated B7-1 and B7-2 expression on nonobese diabetic mouse B cells is associated with increased T cell costimulation and the development of insulitis. J Immunol. 2005;174(2):680-687.

59. Querol L, et al. Rituximab in treatment-resistant CIDP with antibodies against paranodal proteins. Neurol Neuroimmunol Neuroinflamm. 2015;2(5):e149.

60. Kiefer R, Kieseier BC, Brück W, Hartung HP, Toyka KV. Macrophage differentiation antigens in acute and chronic autoimmune polyneuropathies. Brain. 1998;121(Pt 3):469-479.

61. Allard DE, et al. Schwann cell-derived periostin promotes autoimmune peripheral polyneuropathy via macrophage recruitment. J Clin Invest. 2018;128(10):4727-4741.

62. Stratton JA, et al. Macrophages regulate schwann cell maturation after nerve injury. Cell Rep. 2018;24(10):2561-2572.e6.

63. Jessen KR, Mirsky R, Lloyd AC. Schwann cells: development and role in nerve repair. Cold Spring Harb Perspect Biol. 2015;7(7):a020487.

64. Kim HA, Mindos T, Parkinson DB. Plastic fantastic: Schwann cells and repair of the peripheral nervous system. Stem Cells Transl Med. 2013;2(8):553-557.

65. Bosse F, Küry P, Müller HW. Gene expression profiling and molecular aspects in peripheral nerve regeneration. Restor Neurol Neurosci. 2001;19(1-2):5-18.

66. Mao S, et al. Alternative RNA splicing associated with axon regeneration after rat peripheral nerve injury. Exp Neurol. 2018;308:80-89.

67. Song H, et al. MIF/CD74 axis participates in inflammatory activation of Schwann cells following sciatic nerve injury. $J$ Mol Histol. 2019;50(4):355-367.

68. Dong C, Greathouse KM, Beacham RL, Palladino SP, Helton ES, Ubogu EE. Fibronectin connecting segment-1 peptide inhibits pathogenic leukocyte trafficking and inflammatory demyelination in experimental models of chronic inflammatory demyelinating polyradiculoneuropathy. Exp Neurol. 2017;292:35-45.

69. Schmidt B, Toyka KV, Kiefer R, Full J, Hartung HP, Pollard J. Inflammatory infiltrates in sural nerve biopsies in Guillain-Barre syndrome and chronic inflammatory demyelinating neuropathy. Muscle Nerve. 1996;19(4):474-487.

70. Sommer C, Koch S, Lammens M, Gabreels-Festen A, Stoll G, Toyka KV. Macrophage clustering as a diagnostic marker in sural nerve biopsies of patients with CIDP. Neurology. 2005;65(12):1924-1929.

71. Mathey EK, et al. Chronic inflammatory demyelinating polyradiculoneuropathy: from pathology to phenotype. J Neurol Neurosurg Psychiatry. 2015;86(9):973-985.

72. Staudt M, Diederich JM, Meisel C, Meisel A, Klehmet J. Differences in peripheral myelin antigen-specific T cell responses and T memory subsets in atypical versus typical CIDP. BMC Neurol. 2017;17(1):81.

73. D'Antonio M, et al. Gene profiling and bioinformatic analysis of Schwann cell embryonic development and myelination. Glia. 2006;53(5):501-515.

74. Renaud S, et al. Gene expression profiling in chronic inflammatory demyelinating polyneuropathy. J Neuroimmunol. 2005;159(1-2):203-214.

75. Steck AJ, Kinter J, Renaud S. Differential gene expression in nerve biopsies of inflammatory neuropathies. J Peripher Nerv Syst. 2011;16 Suppl 1:30-33.

76. Lee G, Xiang Z, Brannagan TH, Chin RL, Latov N. Differential gene expression in chronic inflammatory demyelinating poly- 
neuropathy (CIDP) skin biopsies. J Neurol Sci. 2010;290(1-2):115-122.

77. Richard A, et al. Transcriptome analysis of peripheral blood in chronic inflammatory demyelinating polyradiculoneuropathy patients identifies TNFR1 and TLR pathways in the IVIg response. Medicine (Baltimore). 2016;95(19):e3370

78. Shin JE, Ha H, Cho EH, Kim YK, Cho Y. Comparative analysis of the transcriptome of injured nerve segments reveals spatiotemporal responses to neural damage in mice. J Comp Neurol. 2018;526(7):1195-1208.

79. Hong YB, Jung SC, Lee J, Moon HS, Chung KW, Choi BO. Dynamic transcriptional events in distal sural nerve revealed by transcriptome analysis. Exp Neurobiol. 2014;23(2):169-172.

80. Uttam S, et al. Translational profiling of dorsal root ganglia and spinal cord in a mouse model of neuropathic pain. Neurobiol Pain. 2018;4:35-44.

81. Sapio MR, Goswami SC, Gross JR, Mannes AJ, Iadarola MJ. Transcriptomic analyses of genes and tissues in inherited sensory neuropathies. Exp Neurol. 2016;283(Pt A):375-395

82. Palladino SP, et al. The human blood-nerve barrier transcriptome. Sci Rep. 2017;7(1):17477

83. Klein AM, et al. Droplet barcoding for single-cell transcriptomics applied to embryonic stem cells. Cell. 2015;161(5):1187-1201.

84. Macosko EZ, et al. Highly parallel genome-wide expression profiling of individual cells using nanoliter droplets. Cell. 2015;161(5):1202-1214

85. Picelli S, Faridani OR, Björklund AK, Winberg G, Sagasser S, Sandberg R. Full-length RNA-seq from single cells using Smartseq2. Nat Protoc. 2014;9(1):171-181.

86. Zheng GX, et al. Massively parallel digital transcriptional profiling of single cells. Nat Commun. 2017;8:14049.

87. Islam S, et al. Quantitative single-cell RNA-seq with unique molecular identifiers. Nat Methods. 2014;11(2):163-166.

88. Hu G, et al. Single-cell RNA-seq reveals distinct injury responses in different types of DRG sensory neurons. Sci Rep. 2016;6:31851

89. Jäkel S, et al. Altered human oligodendrocyte heterogeneity in multiple sclerosis. Nature. 2019;566(7745):543-547

90. Usoskin D, et al. Unbiased classification of sensory neuron types by large-scale single-cell RNA sequencing. Nat Neurosci. 2015;18(1):145-153

91. Carr MJ, et al. Mesenchymal precursor cells in adult nerves contribute to mammalian tissue repair and regeneration. Cell Stem Cell. 2019;24(2):240-256.e9.

92. Meyer zu Hörste G, Hartung HP, Kieseier BC. From bench to bedside--experimental rationale for immune-specific therapies in the inflamed peripheral nerve. Nat Clin Pract Neurol. 2007;3(4):198-211.

93. Ydens E, et al. Acute injury in the peripheral nervous system triggers an alternative macrophage response. J Neuroinflammation 2012;9:176.

94. Allen JA. Chronic demyelinating polyneuropathies. Continuum (Minneap Minn). 2017;23(5):1310-1331.

95. Gawel DR, et al. A validated single-cell-based strategy to identify diagnostic and therapeutic targets in complex diseases. Genome Med. 2019;11(1):47.

96. Kim KT, et al. Application of single-cell RNA sequencing in optimizing a combinatorial therapeutic strategy in metastatic rena cell carcinoma. Genome Biol. 2016;17:80

97. Achim K, et al. High-throughput spatial mapping of single-cell RNA-seq data to tissue of origin. Nat Biotechnol. 2015;33(5):503-509.

98. Moffitt JR, Hao J, Wang G, Chen KH, Babcock HP, Zhuang X. High-throughput single-cell gene-expression profiling with multiplexed error-robust fluorescence in situ hybridization. Proc Natl Acad Sci USA. 2016;113(39):11046-11051.

99. Vickovic S, et al. High-definition spatial transcriptomics for in situ tissue profiling. Nat Methods. 2019;16(10):987-990.

100. Satija R, Farrell JA, Gennert D, Schier AF, Regev A. Spatial reconstruction of single-cell gene expression data. Nat Biotechnol. 2015;33(5):495-502.

101. Ståhl PL, et al. Visualization and analysis of gene expression in tissue sections by spatial transcriptomics. Science. 2016;353(6294):78-82.

102. Salmén F, et al. Barcoded solid-phase RNA capture for Spatial Transcriptomics profiling in mammalian tissue sections. Nat Pro toc. 2018;13(11):2501-2534

103. Codeluppi S, et al. Spatial organization of the somatosensory cortex revealed by osmFISH. Nat Methods. 2018;15(11):932-935

104. Maniatis S, et al. Spatiotemporal dynamics of molecular pathology in amyotrophic lateral sclerosis. Science. 2019;364(6435):89-93.

105. Lein E, Borm LE, Linnarsson S. The promise of spatial transcriptomics for neuroscience in the era of molecular cell typing. Science. 2017;358(6359):64-69. 